\title{
Functional Outcomes after Corrective Osteotomy of Symptomatic Distal Radius Malunions in Children
}

\author{
$\begin{array}{llll}\text { C. A. Selles, } M D^{1,2} & \text { M. A. M. Mulders, MD, } \mathrm{PhD}^{1} & \text { G. R. Roukema, MD } & \text { C. H. van der Vlies, MD, } \mathrm{PhD}^{1}\end{array}$ \\ B. I. Cleffken, $\mathrm{MD}^{1,2}$ \\ M. H. J. Verhofstad, MD, $\mathrm{PhD}^{3}$ \\ N. W. L. Schep, MD, PhD, MSc ${ }^{1,2}$
}

${ }^{1}$ Department of Trauma, Maasstad Hospital, Rotterdam, The Netherlands

${ }^{2}$ Department of Hand Surgery, Maasstad Hospital, Rotterdam, The

Address for correspondence C. A. Selles, MD, Department of Trauma Surgery, Maasstad Hospital, PO Box 9100, 3007 AC Rotterdam, Netherlands

${ }^{3}$ Trauma Research Unit, Department of Surgery, Erasmus University The Netherlands (e-mail: sellesc@maasstadziekenhuis.nl).

Medical Center, Rotterdam, The Netherlands

J Wrist Surg 2020;9:136-140.

\begin{abstract}
Background Closed reduction and cast immobilization of displaced distal radius fractures carries the risk of secondary displacement, which could result in a symptomatic malunion. In patients with a symptomatic malunion, a corrective osteotomy can be performed to improve pain and functional impairment of the wrist joint.

Objective The aim of this study was to assess the functional outcomes of children who underwent a corrective osteotomy due to a symptomatic malunion of the distal radius.

Methods Between 2009 and 2016, all consecutive corrective osteotomies of the distal radius of patients younger than 18 years were reviewed. The primary outcome was functional outcome assessed with the ABILHAND-Kids score. Secondary outcomes were QuickDASH (Quick Disabilities of Arm, Shoulder, and Hand) score, range of motion, complications, and radiological outcomes.

Results A total of 13 patients with a median age of 13 years (interquartile range [IQR]: 12.5-16) were included. The median time to follow-up was 31 months (IQR: 26-51). The median ABILHAND-Kids score was 42 (range: 37-42), and the median QuickDASH was 0 (range: 0-39). Range of motion did not differ significantly between the injured and the

Keywords

- distal radius

- malunion

- corrective osteotomy

- pediatric

- functional outcomes uninjured sides for all parameters. One patient had a nonunion requiring additional operative treatment. The postoperative radiological parameters showed an improvement of radial inclination, radial height, ulnar variance, dorsal tilt, and dorsal tilt.

Conclusion Corrective osteotomy for children is an effective method for treating symptomatic malunions of the distal radius.

Level of Evidence This is a Level IV study.
\end{abstract}

Displaced fractures of the distal radius in children are usually managed by closed reduction and cast immobilization, whereas unstable fractures are mostly fixed with Kirschner wires (K-wires). However, these treatment modalities may lead to secondary displacement, which, in turn, may lead to a

received

August 22, 2019

accepted after revision

November 13, 2019

published online

December 30, 2019 symptomatic malunion of the distal radius. A malunion of the distal radius can become symptomatic causing pain, weakness, or functional impairment of the wrist joint. ${ }^{1,2}$

Various types of corrective osteotomies have been developed for treating distal radius malunions. An open wedge
Copyright $\odot 2020$ by Thieme Medical Publishers, Inc., 333 Seventh Avenue, New York, NY 10001, USA Tel: +1(212) 760-0888.
DOI https://doi.org/ 10.1055/s-0039-3402082. ISSN 2163-3916. 
osteotomy is a technique that can be performed to correct radial length, radial inclination, and rotation. ${ }^{3}$ Open wedge osteotomy is an established technique in adult patients. ${ }^{4} \mathrm{~A}$ fixed-angle plate can be used as a lever device to correct the deformity. ${ }^{5}$ When there is only radial shortening, simple radius lengthening will suffice.

Functional outcomes measured with patient-rated outcome measures have been performed for adult patients undergoing a corrective osteotomy. ${ }^{4}$ However, a few studies have been performed on corrective osteotomies in children. The existing studies usually report on radiological parameters and not on functional outcomes of these patients. ${ }^{6,7}$ Functional outcomes may help clinicians in their decisionmaking as well as in informing patients and their parents about the outcomes prior to surgery. Therefore, the aim of this study is to assess the functional outcomes in children who underwent a corrective osteotomy due to a symptomatic malunion of the distal radius.

\section{Methods}

In this retrospective cohort study, all consecutive pediatric (under 18 years of age) patients who underwent a corrective osteotomy for a symptomatic distal radius malunion between 2009 and 2016 were evaluated. Patients undergoing a corrective osteotomy due to a malunion of the distal radius for any other reason than a previous trauma, such as Kienbock's disease, were excluded.

Malunion, as measured on plain radiographs, for fullgrown patients or those 16 years and older was defined as radial inclination $\leq 15$ degrees, loss of radial height $\geq 5 \mathrm{~mm}$, dorsal angulation $\geq 15$ degrees, and palmar angulation $\geq$ 20 degrees. $^{8}$ For patients between 6 and 16 years of age, dorsal angulation of more than 20 degrees and palmar angulation of more than 25 degrees was considered a malunion. In patients up to 6 years of age, any degree of angulation was accepted unless this was symptomatic. Moreover, malunion was also defined as more than $2-\mathrm{mm}$ positive ulnar variance, one-third shaft displacement, malrotation, and DRUJ incongruency. All patients had to have symptomatic malunions.

The primary outcome was the ABILHAND-Kids score. This questionnaire, filled in by parents with their children, is a measure of the manual ability for children with upper limb impairments. The scale is validated for children with cerebral palsy. The scale ranges from 0 to 42 , with 42 indicating a maximum score in a child's ability to manage daily activities that require the use of the upper limb. ${ }^{9}$ Secondary outcomes were the Quick Disability of the Arm, Shoulder, and Hand (QuickDASH) score, range of motion, and grip strength compared with the injured wrist, pain as indicated on the visual analog scale (VAS), radiological parameters, time to union, and complications. The QuickDASH is an eleven item questionnaire designed to measure physical function and symptoms in people with a musculoskeletal disorder of the upper limb, valid for older children and adolescents. ${ }^{10}$ The QuickDASH ranges from 0 to 100 , with 0 indicating no pain and no functional impairment. ${ }^{11,12}$

\section{Surgical Technique}

All corrective osteotomies were performed by two highly experienced surgeons as classified by Tang. ${ }^{13}$ Antibiotic prophylaxis (cefazolin $30 \mathrm{mg} / \mathrm{kg}$ ) was given preoperatively. The Henry volar approach was used in all patients. The radius was exposed and the appropriate level of the osteotomy was determined, after which an open wedge or lengthening osteotomy was performed. A graft was used if more than $1 \mathrm{~cm}$ of lengthening was necessary. The grafts consisted of demineralized bone matrix (DBM), autogenic grafts, and allogenic hipbone grafts. Ulnar epiphysiodesis was performed to prevent progressive ulnar growth after radial lengthening of a radius with closed physis was performed. Finally, the fracture was fixated with an angular stable volar plate.

\section{Functional and Radiographic Evaluation}

Institutional Review Board approval was obtained by the ethics committee and institutional board of our hospital. All patients who had undergone a corrective osteotomy at least 1 year ago were invited to visit the outpatient clinic to fill out the questionnaires and undergo physical examination. The patients, with their parents when necessary, filled out the ABILHANDKids and QuickDASH questionnaire. The ABILHAND-Kids questionnaire is a measure of the manual ability for children with upper limb impairments. The scale is validated for cerebral palsy children and measures a child's ability to manage daily activities that require the use of the upper limbs. Children and their parents are provided 21 questions on the perceived difficulty of various activities on a three-level scale: impossible, difficult, and easy. The maximum score is 42 , indicating no difficulty in managing daily activities. ${ }^{9}$

The QuickDASH is a shortened version of the DASH with 11 items. The score ranges from 0 to 100 , with 0 indicating no disability. ${ }^{14}$ In addition, patients were asked to indicate their pain as on a VAS score pre- and postoperatively and were asked if their symptoms improved after the corrective osteotomy.

Range of motion was measured using a goniometer and included radial and ulnar deviation, pronation and supination, and dorsal and palmar flexion. Grip strength was measured using a Baseline Hydraulic Hand Dynamometer (Fabrications Enterprises Inc., White Plains, NY). This was performed three times, and an average of these measurements was used. Both range of motion and grip strength were measured for both the injured and uninjured sides.

Radiographic evaluation was performed pre- and postoperatively on standard lateral and posteroanterior radiographs and included radial inclination, radial height, ulnar variance, and dorsal and palmar angulation.

Time to union was defined as the time between the corrective osteotomy and bridging of the fracture site by callus.

\section{Statistical Analysis}

General descriptive statistics on patient characteristics at baseline were performed, including factors such as gender and age, and presented as percentages (categorical variables), means, and standard deviation (continuous variables, normally distributed), or median and interquartile range 
Table 1 Patient characteristics

\begin{tabular}{|c|c|}
\hline & Number (\%) \\
\hline Age at corrective osteotomy, median (IQR) & $13(12.5-16)$ \\
\hline Number of males & $11(85)$ \\
\hline Fracture of dominant wrist & $3(23)$ \\
\hline \multicolumn{2}{|l|}{ Trauma mechanism } \\
\hline Fall from standing height & $2(15)$ \\
\hline Sports related & $11(85)$ \\
\hline
\end{tabular}

Abbreviation: IQR, interquartile range.

(continuous variables, not normally distributed), whichever applicable.

The difference in range of motion, grip strength, radiological parameters, and pain pre- and postoperatively was analyzed using a paired Student's $t$-test. Values of $p<0.05$ were considered significant.

\section{Results}

A total of 13 patients with a corrective osteotomy were included in this cohort. In this period, a total of 2,027 pediatric patients were treated for a distal radius fracture in our institution. Patients had a median age of 13 (interquartile range [IQR]: 12.5-16), and 11 were males. The median time to follow-up was 31 months (IQR: 26-51). Corrective osteotomy was performed within a median of 18 weeks (IQR: 2-62) after initial trauma. Initial treatment consisted of plaster cast immobilization $(n=9)$, K-wire fixation $(n=3)$, and volar plate fixation $(n=1)$. Patient characteristics are presented in -Table 1.

An open wedge osteotomy was performed in 11 patients (-Fig. 1). In two patients, a radius lengthening with an additional ulnar epiphysiodesis was performed (-Fig. 2). Grafts were used in six patients, of which two were allogenic hip grafts, two were autogenic grafts (one greater trochanter and one ulna graft), and two were DBM.

Out of the 13 patients invited for follow-up, 11 completed the questionnaires and the clinical evaluation, and 1 patient completed only the questionnaires. One patient was lost to

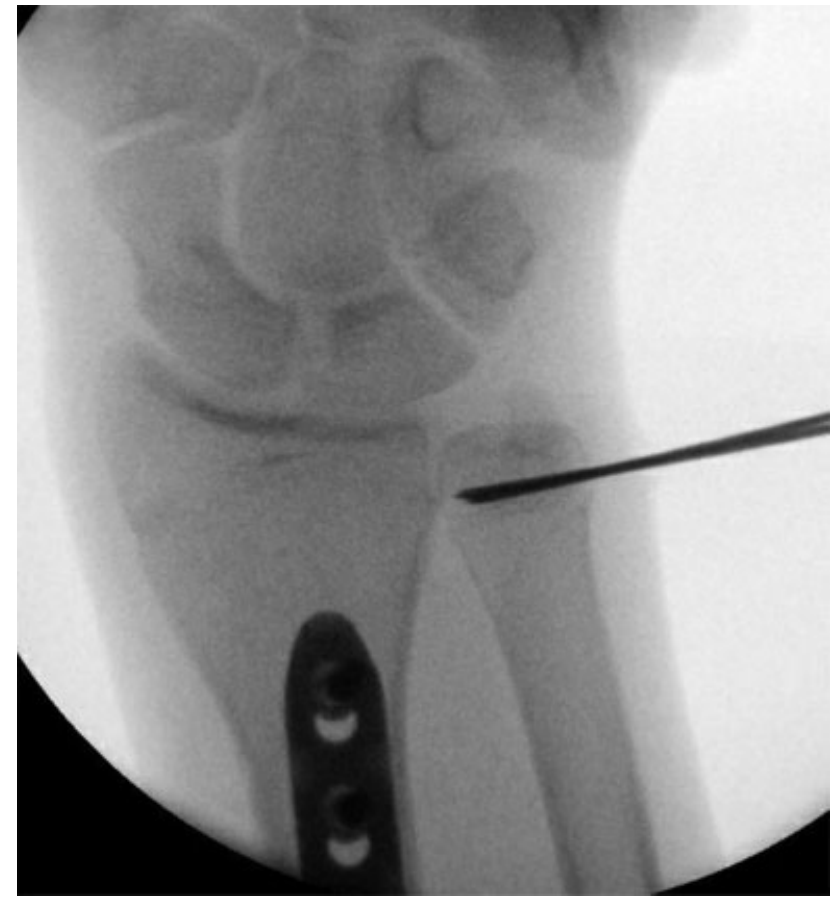

Fig. 2 Ulnar epiphysiodesis.

follow-up. The median ABILHAND-Kids score was 42 (IQR: 42-42), with a range of 37 to 42 , and median the QuickDASH was 0 (IQR: $0-7$ ), with a range of 0 to 39 . The median preoperative VAS score was 6 (IQR: 2.3-7), which improved significantly to 0 postoperatively (IQR: $0-0)(p=0.003)$. Preoperative complaints were pain $(n=9)$, pain and loss of function $(n=2)$, and loss of function $(n=1)$. All but one patient had an improvement in their complaints after the corrective osteotomy. One patient complained of persistent pain, which was due to the plate. The patient was planned for plate removal, after which his pain improved from a VAS score of 3 to 0 , measured 2 weeks postoperatively.

Range of motion did not differ significantly between the injured and the uninjured sides for all parameters ( - Table 2 ). Grip strength in the injured side was significantly lower $(87 \%$ of the uninjured side) $(p=0.04)$.

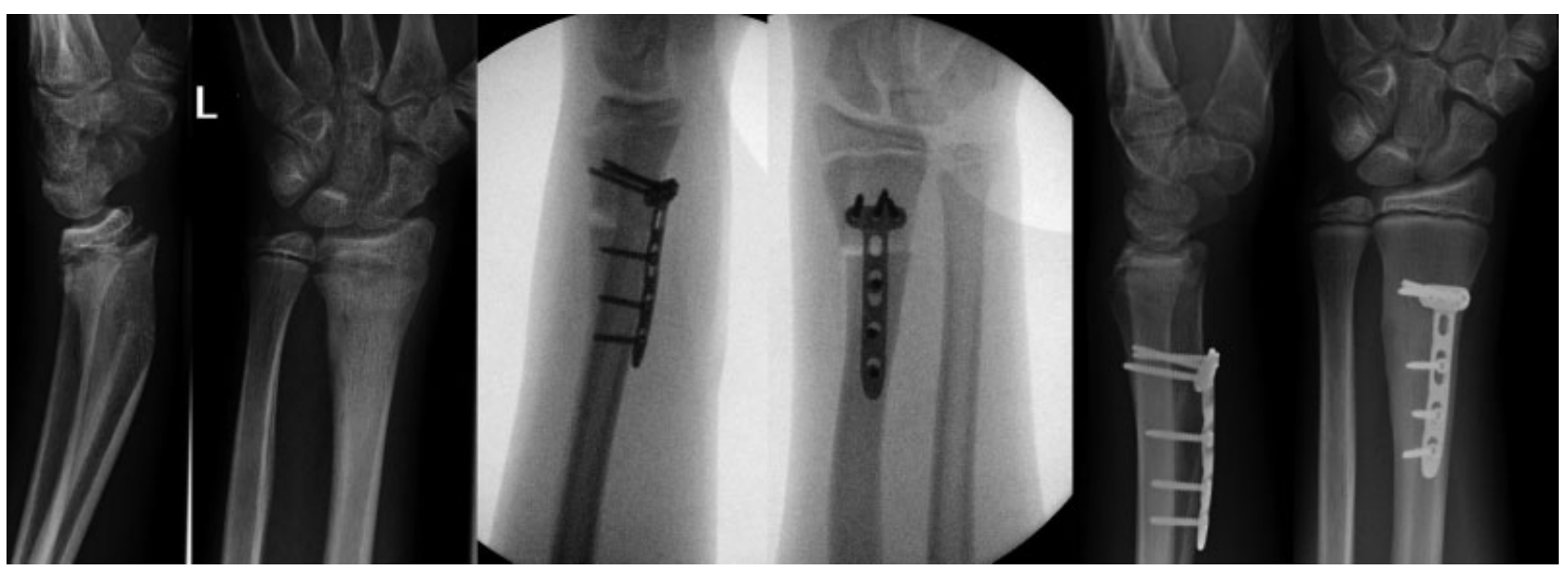

Fig. 1 Open wedge osteotomy: pre- and postoperative radiographs. L, left. 
Table 2 Range of motion and grip strength at follow-up

\begin{tabular}{|l|l|l|l|l|}
\hline $\begin{array}{l}\text { Range of } \\
\text { motion, } \\
\text { grip strength, } \\
\text { mean (SD) }\end{array}$ & $\begin{array}{l}\text { Injured } \\
\text { side }\end{array}$ & $\begin{array}{l}\text { Uninjured } \\
\text { side }\end{array}$ & Difference & $p$-Value \\
\hline $\begin{array}{l}\text { Radial } \\
\text { deviation }\end{array}$ & $16(4)$ & $17(3)$ & $0.5(2)$ & 0.34 \\
\hline $\begin{array}{l}\text { Ulnar } \\
\text { deviation }\end{array}$ & $22(3)$ & $21(3)$ & 0 & - \\
\hline Pronation & $87(4)$ & $88(3)$ & $0.5(2)$ & 0.34 \\
\hline Supination & $87(3)$ & $87(3)$ & 0 & - \\
\hline Dorsal flexion & $84(6)$ & $84(4)$ & 0 & - \\
\hline Volar flexion & $84(5)$ & $84(4)$ & $0.5(2)$ & 0.34 \\
\hline $\begin{array}{l}\text { Grip } \\
\text { strength (kg) }\end{array}$ & $27(8)$ & $31(11)$ & $3(5)$ & 0.04 \\
\hline
\end{tabular}

Abbreviation: SD, standard deviation.

Note: Values in bold are significant $(p<0.05)$.

One patient, a 13-year-old girl, required additional surgery due to a nonunion following open wedge osteotomy. She was successfully treated with an autograft 8 months later. Nine patients had their plate removed due to symptomatic hardware.

The radiological parameters after corrective osteotomy compared with those before corrective osteotomy showed an improvement of the radial inclination, radial height, ulnar variance, and dorsal tilt (-Table 3 ). The median time to union was 3 months (IQR: $1-5.5$ ).

\section{Discussion}

Our results show that corrective osteotomy of the distal radius for a symptomatic malunion in children provides good functional outcomes. Range of motion of the injured wrist is comparable to that of the uninjured wrist. Furthermore, pain scores decreased and radiographic parameters improved significantly after the corrective osteotomy.

Satisfactory results for corrective osteotomies for malunited fractures of the forearm in children have previously been reported. ${ }^{15}$ This study did not, however, focus specifically on distal radius malunions but also analyzed fractures of both the radius and ulna, as well as distal and midshaft fractures. In our study, we found a median ABILHAND-Kids score of 42 and a median QuickDASH of 0. These patient-rated functional out- comes cannot be compared with results in the existing literature, as no functional outcomes for children have yet been described. Previous studies showed a DASH score of 10 to 16 after corrective osteotomy in adult patients. ${ }^{4,16}$ Another study reported satisfactory outcomes after corrective osteotomy in young and middle-aged patients, with DASH scores ranging from 25 to $33,{ }^{7}$ but these scores are arguably higher than the QuickDASH score found in our study.

Van Geenen and Besselaar reported a significant improvement of range of motion in a cohort of children with a malunion of the forearm. Patients included had fractures of either the radius or the radius and ulna, and location of the fracture varied from proximal and middle to the distal one-third of the bone. ${ }^{17}$ The authors also suggest that the corrective osteotomy should be performed within 1 year, resulting in greater gain in range of motion. Hove and Engesaeter assessed the outcome of six pediatric patients with corrective osteotomies due to malunions of the distal radius. ${ }^{6}$ The authors found complete postoperative pain relief, and a total range of motion that was 96\% compared with the uninjured side.

One of the possible complications of an open wedge osteotomy is a nonunion. ${ }^{3}$ We found one case of nonunion in this cohort. After 7 months, no bridging of the osteotomy site was visible. The patient had no complaints of the malunion and had a full range of motion. The defect was treated with an ulnar cancellous bone graft mixed with autograft from the iliac crest and Cerasorb (Curasan, Research Triangle Park, NC). Three months later, union was achieved. A previously published cohort of corrective osteotomies after injuries of the distal radial physis in children reported no complications, but this was a cohort of only six patients. ${ }^{6}$

There are several limitations to this study. Due to its retrospective nature, no presurgical data were available to compare the functional outcomes with the postoperative ones. The range of motion and grip strength were evaluated at follow-up, but as preoperative data were not available, the improvement could not be measured. These parameters were, however, compared with the uninjured side to give an indication of what the patient's baseline range of motion and grip strength would be. The preoperative VAS was also determined retrospectively, which might lead to recall bias. Future research should focus on collecting presurgical data prospectively to more thoroughly analyze the effect of corrective osteotomy on functional outcome. Furthermore, the use of functional outcomes has become increasingly popular.

Table 3 Radiological evaluation

\begin{tabular}{|l|l|l|l|l|}
\hline Radiological parameter, mean (SD) & Preoperative & Postoperative & Difference & $p$-Value \\
\hline Radial inclination & $18(4)$ & $21(3)$ & $3(3)$ & 0.003 \\
\hline Radial height & $8(2)$ & $9(2)$ & $1(2)$ & 0.046 \\
\hline Ulnar variance & $0.5(3)$ & $-2^{1}$ & $2(2)$ & 0.006 \\
\hline Dorsal tilt & $17(16)$ & $2(3)$ & $4(16)$ & $\mathbf{2 ( 7 )}$ \\
\hline Volar tilt & $6(9)$ & $4(4)$ & 0.004 \\
\hline
\end{tabular}

Abbreviation: SD, standard deviation.

Note: Values in bold are significant $(p<0.05)$. 
However, there are currently no validated questionnaires for children with upper limb trauma. The ABILDHAND-Kids questionnaire is a validated questionnaire for children with cerebral palsy and has been previously used as the next best available outcome measure. ${ }^{18}$ The QuickDASH is a valid instrument for older children and adolescents with upper extremity pathology. ${ }^{10}$ The questionnaires used in this study were the next best available for measuring functional outcomes in a pediatric population. The results provide an insight into the effect of corrective osteotomies for distal radius malunions and valuable information for shared decision-making. Time to union was determined on plain radiographs. The moment the radiograph was taken varied in time between patients. The union could have therefore been achieved earlier than the radiograph was taken.

\section{Conclusion}

Corrective osteotomy is an effective method to treat symptomatic malunions of the distal radius in children with good functional outcomes.

\section{Note}

This work was performed in the Maasstad Hospital.

\section{Funding}

None.

\section{Conflict of Interest \\ None declared.}

\section{References}

1 Graham TJ. Surgical correction of malunited fractures of the distal radius. J Am Acad Orthop Surg 1997;5(05):270-281

2 Slagel BE, Luenam S, Pichora DR. Management of post-traumatic malunion of fractures of the distal radius. Orthop Clin North Am 2007;38(02):203-216, vi

3 Bushnell BD, Bynum DK. Malunion of the distal radius. J Am Acad Orthop Surg 2007;15(01):27-40

4 Mulders MA, d'Ailly PN, Cleffken BI, Schep NW. Corrective osteotomy is an effective method of treating distal radius malunions with good long-term functional results. Injury 2017;48(03):731-737
5 Kaempf de Oliveira R, Serrano PJ, Badia A, Ferreira MT. Corrective osteotomy after damage of the distal radial physis in children: surgical technique and results. Tech Hand Up Extrem Surg 2011; 15(04):236-242

6 Hove LM, Engesaeter LB. Corrective osteotomies after injuries of the distal radial physis in children. J Hand Surg [Br] 1997;22(06): 699-704

7 Van Cauwelaert de Wyels J, De Smet L. Corrective osteotomy for malunion of the distal radius in young and middle-aged patients: an outcome study. Chir Main 2003;22(02):84-89

8 Dutch Society for Surgery. Guideline Distal Radius Fractures, diagnosis and treatment 2010. Available at: http://www.heelkunde. nl/uploads/h8/VM/h8VMOIRX83oW2NSR_5rTWw/Richtlijn_ Distale_radius_fracturen_definitieve_versie_0511.pdf. Accessed July 1, 2019

9 Arnould C, Penta M, Renders A, Thonnard JL. ABILHAND-Kids: a measure of manual ability in children with cerebral palsy. Neurology 2004;63(06):1045-1052

10 Quatman-Yates CC, Gupta R, Paterno MV, Schmitt LC, Quatman CE, Ittenbach RF. Internal consistency and validity of the QuickDASH instrument for upper extremity injuries in older children. J Pediatr Orthop 2013;33(08):838-842

11 Beaton DE, Wright JG, Katz JN; Upper Extremity Collaborative Group. Development of the QuickDASH: comparison of three item-reduction approaches. J Bone Joint Surg Am 2005;87(05):1038-1046

12 Kennedy CA, Beaton DE. A user's survey of the clinical application and content validity of the DASH (Disabilities of the Arm, Shoulder and Hand) outcome measure. J Hand Ther 2017;30(01):30-40.e2

13 Tang JB. Re: levels of experience of surgeons in clinical studies. J Hand Surg Eur Vol 2009;34(01):137-138

14 Kennedy CABD, Solway S, McConnell S, Bombardier C. Disabilities of the Arm, Shoulder and Hand (DASH). The DASH and QuickDASH Outcome Measure User's Manual. 3rd ed. Toronto, Ontario: Institute for Work \& Health; 2011

15 Trousdale RT, Linscheid RL. Operative treatment of malunited fractures of the forearm.J Bone Joint Surg Am 1995;77(06):894-902

16 Lozano-Calderón SA, Brouwer KM, Doornberg JN, Goslings JC, Kloen P, Jupiter JB. Long-term outcomes of corrective osteotomy for the treatment of distal radius malunion. J Hand Surg Eur Vol 2010;35(05):370-380

17 van Geenen RC, Besselaar PP. Outcome after corrective osteotomy for malunited fractures of the forearm sustained in childhood. J Bone Joint Surg Br 2007;89(02):236-239

18 Colaris JW, Reijman M, Allema JH, et al. Early conversion to belowelbow cast for non-reduced diaphyseal both-bone forearm fractures in children is safe: preliminary results of a multicentre randomised controlled trial. Arch Orthop Trauma Surg 2013;133 (10):1407-1414 